\title{
Intertextualidad y multimodalidad en constelaciones transmedia: una propuesta interdisciplinar en la formación docente
}

\section{Intertextuality and multimodality in transmedia constellations: an interdisciplinary proposal in teacher training}

\section{José Rovira-Collado}

Universidad de Alicante

jrovira.collado@gcloud.ua.es

ORCID ID: https://orcid.org/0000-0002-3491-8747

\section{Mónica Ruiz-Bañuls}

Universidad de Alicante

monica.ruiz@ua.es

ORCID ID: https://orcid.org/0000-0003-4252-9888

\section{Francisco Antonio Martínez-Carratalá}

Universidad de Alicante

famc@alu.ua.es

ORCID ID: https://orcid.org/0000-0003-4666-5035

\section{Isabel Gómez-Trigueros}

Universidad de Alicante

isabel.gomez@ua.es

ORCID ID: https://orcid.org/0000-0002-0587-5063

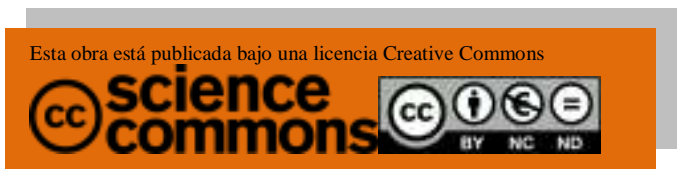

DOI: $10.17398 / 1988-8430.34 .111$

Fecha de recepción: 10/03/2021 Fecha de aceptación: 14/04/2021

Rovira-Collado, J.; Ruiz-Bañuls, M.; Martínez-Carratalá, F., y Gómez-Trigueros, I (2021). Intertextualidad y multimodalidad en constelaciones transmedia: una propuesta interdisciplinar en la formación docente. Tejuelo, 34, 111-142.

Doi: https://doi.org/10.17398/1988-8430.34.111 
Resumen: En la siguiente investigación se propone la evolución de la práctica de las constelaciones literarias a través de los conceptos de multimodalidad y narrativas transmedia. Se realiza un análisis cuantitativo de dos experiencias concretas realizadas durante el curso académico 2019-2020 en la Universidad de Alicante, con el objetivo principal de desarrollar el concepto de intertextualidad entre nuestro alumnado. Se trabajan las constelaciones desde una perspectiva interdisciplinar, donde el uso de la tecnología es ineludible, para la publicación en abierto de las actividades a través de vídeos, presentaciones y blogs. Se han desarrollado diversos cuestionarios mixtos, con una fiabilidad adecuada, para comprobar los aprendizajes de la propuesta, con unos resultados excelentes. La temática de las constelaciones ha variado entre ambas experiencias, desde los pecados capitales a los personajes infantiles tradicionales, demostrando que las constelaciones pueden aplicarse con diversas temáticas y distintos objetivos educativos. La expansión transmedia de la práctica además ha permitido adaptar las constelaciones a los nuevos tiempos de docencia híbrida u online.

Palabras clave: educación literaria; narrativas transmedia; lectura multimodal; hipertextualidad; intertextualidad.

\begin{abstract}
The following research proposes an evolution of the practice of literary constellations through the concepts of multimodality and transmedia narratives. A quantitative analysis of two specific experiences carried out during the academic year 2019-2020 at the University of Alicante is carried out, which confirm the possibilities of this practice in teacher training, with the main objective of developing the concept of intertextuality among our students. The constellations are worked on from an interdisciplinary perspective, where the use of technology is unavoidable, for the open publication of activities through videos, presentations and blogs. Various mixed questionnaires have been developed, with adequate reliability, to verify the learning of the proposal, with excellent results. The theme of the constellations has varied between the two experiences, from the deadly sins to traditional children's characters, showing that the constellations can be applied for varied themes and different educational purposes. The transmedia expansion of the practice has also made it possible to adapt the constellations to the new times of hybrid or online teaching.
\end{abstract}

Keywords: literary education, transmedia narratives, multimodal reading, hypertextuality, intertextuality. 


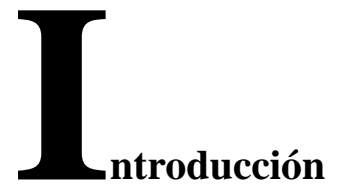

Las constelaciones literarias son un formato visual para organizar una serie de lecturas con un elemento temático en común, a través de la representación gráfica de una serie de estrellas, nexos o puntos enlazados (Jover, 2009). La organización de dichos esquemas puede articularse en torno a una época, temática o género literario, ofreciendo múltiples formas de representación. Dicho formato puede aplicarse en los diferentes niveles educativos para mostrar distintos contenidos y puede considerarse apropiado para el desarrollo de la Educación Literaria en distintos niveles educativos. Desde la Universidad de Alicante (Rovira-Collado, 2019), hemos querido ampliar sus posibilidades para relacionarlas con el concepto de 
narrativas transmedia (Jenkins, 2003; Scolari, 2013), permitiendo incluir cualquier tipo de texto multimodal (Kress, 2010; Unsworth, 2014). El objetivo principal de esta investigación es comprobar si la práctica docente de las constelaciones multimodales es efectiva para la comprensión de las relaciones intertextuales.

Las constelaciones multimodales creadas por nuestro alumnado incluyen vídeos, obras de arte, cómics y otros elementos multimodales junto con textos literarios de distintas etapas y géneros. En esta práctica confluyen la creatividad literaria con la multimodalidad (CaroValverde, 2019). Esta investigación se ha implementado durante el curso 2019-2020 en dos asignaturas del área de Didáctica de la Lengua y la Literatura de nuestra universidad, una del Grado de Educación Primaria y otra del Máster en Profesorado de Educación Secundaria Obligatoria y Bachillerato, Formación Profesional y Enseñanza de Idioma. Las experiencias son consecutivas pero la secuencia y el producto creado por el alumnado es casi idéntico y la metodología de la investigación es la misma. Se trata de un estudio de tipo mixto (cuantitativo y cualitativo), con encuestas como instrumento de análisis, así como la observación directa en el aula y blogs para publicar en abierto las constelaciones generadas por el alumnado. Además de la evaluación de la propuesta, se han analizado los elementos más recurrentes de cada constelación. Los resultados confirman el valor formativo de las constelaciones transmedia como práctica docente efectiva para activar el intertexto lector (Mendoza, 2001) del alumnado al tiempo que se propone la ampliación de dicho término por el de intertexto multimodal.

\section{Marco Teórico}

En la formación del futuro profesorado de lengua y literatura en Educación Primaria y Secundaria se hace inevitable el conocimiento y desarrollo de metodologías y recursos específicos que les permitan realmente hacer un uso funcional y formativo del potencial de las nuevas tecnologías relacionadas con la Didáctica de la Lengua y la Literatura, en adelante DLL (Arbonés et al., 2015). Aunque en la etapa 
de Primaria no hay especialistas en lengua y literatura, siendo el profesorado generalista, la formación desde nuestra área debe otorgarles suficientes aprendizajes para la formación de su alumnado como lectores literarios (Ballester, 2015). Actualmente en esta área nos encontramos con la necesidad del desarrollo conjunto de la competencia literaria para mejorar los índices de lectura (Cerrillo, 2007) y la competencia digital docente (Prendes, Gutiérrez \&, Castañeda 2019). Se precisan por ello propuestas e investigaciones para que la educación literaria se adecúe al marco de la educación 2.0 y las textualidades multimodales se transformen en un referente trasversal y multidisciplinar (Mendoza, Castro, Gordillo \& Gómez, 2016) esencial en el nuevo contexto docente.

Podemos señalar, entre los retos de la formación docente, la necesidad de abordar propuestas de innovación y de investigación donde se potencie el trabajo cooperativo (Hernández \& Rovira-Collado, 2020). Proyectos que consoliden procesos de reflexión, procedimientos que susciten y promuevan cambios significativos en la concepción y evaluación de las actividades de enseñanza y aprendizaje (Rebolledo, 2015; Paredes, Guitert \& Rubia, 2015). Nuestra propuesta trata de ampliar la enseñanza de la lectura más allá de la distinción entre textos literarios y no literarios, abriéndonos así al trabajo interdisciplinar entre diversas áreas de conocimiento (Gómez \& Ruiz, 2018).

A la hora de trabajar la formación del lector literario, el papel del futuro profesorado es esencial pues se enfrenta a un triple desafío: enseñar a leer, consolidar hábitos lectores y compartir un imaginario colectivo (Jover, 2009; Romero, Ambrós \& Trujillo, 2020). La aplicación de estrategias didácticas para convertir al alumnado en lectores competentes y autónomos de literatura a lo largo de su vida es una prioridad en la actualidad, en todas las etapas educativas. El profesorado en formación necesita conocer y desarrollar metodologías que integren los procedimientos y recursos de las nuevas tecnologías (Castañeda, 2019) en la educación literaria, con nuevas lecturas digitales (Amo \& Núñez Delgado, 2019). Por lo tanto, planteamos una línea de actuación didáctica en la que, como se ha señalado, el eje central es la ampliación del concepto de constelación literaria hacia la 
multimodalidad digital como práctica docente que vaya más allá de la mera textualidad (Amo, 2019).

Para Garvis (2015), el concepto de constelación apunta a una red o camino estrellado donde el propio lector va enlazando una serie de lecturas que comparten algún tema en común, es decir, "in a story constellation, an individuals narratives of experience relate to each another" (2015, p.52). Según las experiencias personales de cada persona, cada cual irá trazando su selección de obras fruto de su intertexto lector (Mendoza, 2001, 2012). En el ámbito de la DLL, debemos señalar que Guadalupe Jover (2007) propuso la elaboración de constelaciones literarias creadas por el profesor como construcciones interpretativas que permitieran al lector la incursión paulatina y secuenciada, en el territorio de la gran literatura. En resumen, se trata de mostrar los múltiples lazos, nexos o relaciones intertextuales, que unen una obra cualquiera con otras tantas a lo largo de la historia. Jover destaca las múltiples posibilidades para plantear esas relaciones entre unas obras "con otras manifestaciones artísticas del momento, con el entramado cultural e ideológico de la época, con unas determinadas condiciones de vida de autores y lectores, con el circuito de recepción y de producción" (2007, p.119).

Este carácter hipertextual de las constelaciones planteado por Jover (2009) adquiere una doble significación al aplicar la idea de hipertexto digital (Landow, 2009) y del enorme potencial de las tecnologías para un cambio radical del papel del estudiante y del docente en la educación literaria y tecnológica. Es necesario aprender a leer hipertextos (Mendoza, 2012) y esto implica la participación activa en el proceso de lectura, algo que en el actual contexto escolar resulta esencial.

Consideramos que el diseño de constelaciones multimodales que en este estudio se plantea, con sus redes de nodos y enlaces o hipervínculos, como un proyecto transmedia (Hernández \& RoviraCollado, 2020) o de tecnología educativa (De Benito \& Salinas, 2016), permite superar las jerarquías y desplaza al lector la responsabilidad de decidir por dónde seguirá la lectura, en qué aspectos desea profundizar 
o qué otros elementos pasarán por alto. La formación lectora del hipertexto literario (Mendoza \& Romea, 2010) resulta esencial para los jóvenes vinculados a la experimentación digital, a los que, por lo general, solemos ignorar o no atender (Romea, 2008).

Más allá del texto escrito, al proponer la elaboración de nuestras constelaciones, nos encontramos enlazando múltiples formatos que implican una lectura multimodal: desde la interrelación entre texto e imagen del álbum ilustrado y el cómic (Leeuwen \& Jewitt, 2001) a las múltiples posibilidades de lectura en pantalla (Kress, 2010; Unsworth, 2014).

De este modo, las textualidades multimodales se convierten en un referente transversal e interdisciplinar (Arbonés et al., 2015, p.97) esencial en la educación literaria. Estamos asistiendo al desarrollo exponencial de lecturas y escrituras que interfieren, se enriquecen, se solapan y pasan casi desapercibidas en el día a día (Romea, 2008). Si queremos convertir al alumnado universitario en un lector competente de la tradición literaria, su formación requiere metodologías variadas y ajustadas a las capacidades e intereses de los destinatarios. Metodologías que "les ayuden a comprender, para llegar a alguna interpretación del contenido significativo del texto, acorde con su experiencia vital y su cultura personal" (Romea, 2008, p.96). La metodología implementada en la elaboración de las constelaciones muestra un ejemplo de actuación didáctica durante la formación inicial del profesorado, donde además de la hipertextualidad, se reflejan las relaciones intertextuales entre los elementos trabajados (Genette, 2001; Mendoza \& Cerrillo, 2013).

Por ello, al plantear la constelación multimodal como práctica lectora y digital en esta investigación, estamos sosteniendo que el libro de papel, de carácter textual y tangible, está dejando de ser el referente exclusivo en el proceso de enseñanza-aprendizaje. El medio que permite el acceso al conocimiento se está transformando en un ente virtual, digital, de carácter hipertextual (Landow, 2009) o multimodal (Unsworth, 2014). Estas premisas nos permiten introducir en ambas propuestas nuevos conceptos imprescindibles para conocer los 
múltiples soportes a través de los que podemos seguir una narración. El concepto de narrativas transmedia (Jenkins 2003, 2015) ha ido evolucionando (Scolari, 2018) y nos ha permitido incluir en nuestras constelaciones multimodales elementos como videojuegos, ilustraciones, películas, canciones, cómics, series, vídeos en Internet, redes sociales... También la música puede ser una herramienta fundamental para trabajar la intertextualidad (Vicente-Yagüe, 2014).

Las temáticas de las constelaciones creadas en ambas experiencias, los "pecados capitales y los protagonistas de la literatura infantil" tradicional nos permiten un completo desarrollo crítico. La fascinación por estos siete vicios, o los valores de esta narrativa ancestral, vienen a resumir las grandes pulsiones del ser humano, formando al alumnado para conocer la evolución de las diversas realidades artísticas. Este aprendizaje se construye a través de un hipertexto elaborado a partir de múltiples fragmentos multimodales de referencias actuales. Dicho universo transmedia (Ambròs Pallarès, 2020; Hernández Ortega, 2019) quedaría constituido no solo por sus vivencias y sus lecturas sino también por la televisión, el cine, los videojuegos, los cómics, la web... y todas aquellas realidades que el alumnado irá seleccionando de acuerdo con su madurez literaria (Jover, 2007).

\section{Metodología}

\section{1. Objetivos}

Esta investigación interdisciplinar se centra en la valoración de la multimodalidad y de la intertextualidad para el desarrollo de la educación literaria y digital, en el ámbito de la formación inicial del profesorado. El objetivo principal de esta investigación es averiguar si se confirman las posibilidades didácticas de una nueva práctica lectora: la constelación multimodal, a través del análisis cuantitativo de dos experiencias consecutivas. También queremos profundizar en el desarrollo de la competencia digital y de la literaria, proponiendo la ampliación del concepto de intertexto lector hacia una lectura multimodal. Podemos concretar también los siguientes objetivos: 
1. Analizar la pertinencia de la constelación multimodal como secuencia didáctica apropiada para el desarrollo de la competencia literaria y digital.

2. Identificar las relaciones hipertextuales y multimodales referidas incluidas en las distintas constelaciones y señalar su importancia en la formación del profesorado.

3. Desarrollar una competencia digital docente crítica que les permita investigar y crear contenidos empleando el blog, las constelaciones y los relatos literarios y audiovisuales como herramientas formativas.

A través de estos objetivos, la hipótesis que se plantea en esta investigación se formula en los siguientes términos:

Hipótesis nula (Ho). No se produce una mejora en el proceso de formación del alumnado de ambos grupos de la Facultad de Educación al utilizar las constelaciones multimodales.

Hipótesis positiva o alternativa (H1). Se produce una mejora cuantificable en el proceso de formación del alumnado de ambos grupos al emplear una secuencia didáctica basada en la creación de constelaciones multimodales y relatos literarios y audiovisuales.

\section{2. Diseño de la práctica}

Desde la actividad de Constelaciones Literarias lanzada por Guadalupe Jover y el Grupo Guadarrama, con variados ejemplos alojados en https://sites.google.com/view/constelaciones-literarias/, la práctica ha ido evolucionando hacia modelos transmedia o multimodales (Rovira-Collado, 2019). En esta nueva propuesta, podemos confirmar que la secuencia ha crecido, saliendo de una actividad con un grupo concreto, a convertirse en una propuesta entre distintas clases o con la participación de dos universidades (MartínezCarratalá y Hernández Delgado, 2020).

En este sentido, queremos señalar que analizaremos dos propuestas consecutivas realizadas durante el curso 2019-2020 en la Universidad de Alicante. Las Constelaciones multimodales sobre 
pecados capitales, realizadas por alumnado del $4^{\circ}$ curso del Grado de Educación Primaria, alojadas

en https://constelacionesmultimodales4dleua19.blogspot.com/ e identificadas con la sigla 4DLE de la asignatura "Didáctica de la Lectura y la Escritura", y las Constelaciones y relatos multimodales de tradición oral, realizadas por el alumnado del Máster de Profesorado, alojadas en https://ecostransmedia2020.blogspot.com/ e identificadas como INVTIC de la asignatura "Investigación, innovación y uso de las TIC en la Enseñanza de la Lengua y la Literatura”. Este último blog también recoge las constelaciones realizadas por el alumnado del grado de Educación Primaria de la Universidad de Murcia (Martínez-Carratalá \& Hernández Delgado, 2020), pero excluimos esas constelaciones de nuestro análisis, por no haber participado directamente como docentes en el diseño de las mismas.

Aunque el instrumento para el análisis cuantitativo es muy similar, encuestas antes y después de la experiencia, la secuencia didáctica ha ido evolucionando progresivamente. En 4DLE el tema central son los pecados capitales, asignados a cada grupo de trabajo. En INVTIC nos centramos en personajes de la tradición oral de la literatura infantil. En la segunda propuesta, además de la constelación multimodal, que incluye epitextos digitales como booktrailer o booktubers (Lluch, Tabernero-Sala \& Calvo-Valios, 2015; PaladinesParedes \& Aliagas, 2021), que son los ejes de la primera, se añaden tres elementos nuevos: constelación bibliométrica, relato literario y relato audiovisual.

Toda esta nueva secuencia está recogida en el blog de Caperucita Transmedia https://caperucitransmedia.blogspot.com/ (Martínez-Carratalá, 2020), que sirve como guion para toda la experiencia.

En los datos bibliométricos se incluyen todas las referencias de los distintos elementos de una constelación. Estos datos son imprescindibles para revisar una constelación, y aunque en experiencias anteriores se solicitaban, hasta ahora no eran una parte específica de la práctica. Con la creación de distintos relatos audiovisuales y literarios, 
con la premisa fundamental de incluir referencias intertextuales a los elementos de la constelación, se amplían las posibilidades de la práctica y la creatividad de nuestro alumnado.

A continuación, presentamos el cronograma de ambas experiencias:

\section{Tabla 1}

Distribución temporal de las dos experiencias y blogs con constelaciones

\begin{tabular}{|c|c|c|}
\hline Fase & Actividad 4DLE- Pecados capitales & Fecha \\
\hline Blog & \multicolumn{2}{|c|}{ https://constelacionesmultimodales4dleua19.blogspot.com/ } \\
\hline Previa & Creación del blog y diseño del cuestionario & Septiembre 2019 \\
\hline Pretest & Realización del cuestionario inicial & 23/09/2019 \\
\hline Formativa & Desarrollo de la propuesta didáctica & $\begin{array}{c}23 / 09 \mathrm{a} \\
15 / 10 / 2019\end{array}$ \\
\hline Final & Presentación en clase de constelaciones & $16 / 10 / 2019$ \\
\hline \multirow[t]{2}{*}{ Postest } & Realización del cuestionario final & $\begin{array}{c}16 / 10 \mathrm{a} \\
21 / 10 / 2019\end{array}$ \\
\hline & $\begin{array}{lll}\text { Constelaciones } & \text { INVTIC- } & \text { Personajes } \\
\text { tradición infantil }\end{array}$ & \\
\hline Blog & https://ecostransmedia2020.blogspot.com/ & \\
\hline Fase & Actividad & Fecha \\
\hline Previa & Creación del blog y revisión del cuestionario & Enero 2020 \\
\hline Pretest & Realización del cuestionario inicial & $17 / 03 / 2020$ \\
\hline Formativa & Desarrollo de la propuesta didáctica & $17 / 03$ a $27 / 04$ \\
\hline Final & Presentación en clase de constelaciones (online) & $27 / 04 / 2020$ \\
\hline Postest & Realización del cuestionario final & $27 / 04$ a $30 / 04$ \\
\hline
\end{tabular}

Fuente: Elaboración propia

\section{3. Muestra}

Dieron el consentimiento informado para participar en la investigación un total de 172 personas, 138 estudiantes en la primera fase en la asignatura "Didáctica de la Lectura y la Escritura-4DLE" (4" curso Grado en Maestro en Educación Primaria) entre septiembre y octubre de 2019 y 34 de "Investigación, innovación y uso de las TIC en la Enseñanza de la Lengua y la Literatura-INVTIC" (Máster de Formación del Profesorado) durante el segundo semestre, entre marzo y 
mayo de 2020 Podemos considerarlos grupos naturales (McGrath, 1986) organizados según las clases de la Facultad de Educación. Por sexo, encontramos 121 mujeres $(69.45 \%)$ y 51 hombres $(30.05 \%)$, con un rango de edad de entre 22 y 48 años, porcentajes habituales en este ámbito (Argibay, 2009). En la primera experiencia 4DLE hubo un grupo experimental (G1) y un grupo de control (G2), que no realizó la práctica de constelaciones. En INVTIC era un único grupo de clase. En la siguiente tabla encontramos la distribución por edad de los tres grupos.

\section{Tabla 2}

Distribución de los grupos analizados por edad

\begin{tabular}{ccccc}
\hline Edad & $\begin{array}{c}\text { G1 Exp. } \\
\text { 4DLE }\end{array}$ & $\begin{array}{c}\text { G2 Cont. } \\
\text { 4DLE }\end{array}$ & G3 INVTIC & Total \\
\hline Entre 18 y 22 años & 75 & 30 & 6 & 111 \\
Entre 23 y 30 años & 11 & 10 & 25 & 46 \\
Entre 31 y 40 años & 2 & 6 & 1 & 9 \\
Más de 40 años & 0 & 4 & 2 & 6 \\
TOTAL & $\mathbf{8 8}$ & $\mathbf{5 0}$ & $\mathbf{3 4}$ & $\mathbf{1 7 2}$ \\
\hline
\end{tabular}

Fuente: Elaboración propia

\subsection{Instrumentos, procedimiento de recogida y análisis de datos}

En ambas propuestas se ha realizado un diseño metodológico mixto, basado en encuestas, antes y después de realizar la práctica (pretest y postest). Los cuatro cuestionarios han sido diseñados específicamente en ambos experimentos basándonos en las propuestas previas de Larrañaga y Yubero (2015) o Del Moral-Barrigüete y Molina-García (2018), para cuestiones referidas a la lectura en la formación del profesorado y en Gómez-Trigueros, Ruiz-Bañuls y Ortega-Sánchez (2019) para las cuestiones transmedia. Todos los cuestionarios fueron revisados y validados por expertos y expertas en didáctica de la lengua y la literatura, tecnología educativa y metodología de la investigación. Los formularios incluyen el aviso de privacidad y el consentimiento informado de las y los participantes para confirmar su participación desinteresada. 
Se informó a todo el alumnado durante las dos experiencias del objetivo de la investigación, así como de la confidencialidad con que serían tratadas las respuestas emitidas y se les solicitó el citado consentimiento informado para su realización. Para la difusión de los cuestionarios, alojados en los blogs de cada experiencia, se ha utilizado Google Forms. Los dos cuestionarios de la primera fase (4DLE) tienen 23 ítems mixtos, y los dos de la segunda (INVTIC), 18, simplificando el instrumento. Tenemos preguntas con escala Likert de cinco puntos (1, Muy en desacuerdo/Desconocimiento total - 5, Totalmente de acuerdo/Muy conocido) para los datos cuantitativos y otras preguntas abiertas, que generalmente corresponden a la ampliación o comentario de las anteriores preguntas. Además, se recopilan datos sociodemográficos que se utilizan de forma anónima.

El diseño mixto de la investigación nos permite realizar tanto análisis descriptivos como inferenciales, que nos llevan a confirmar los objetivos de esta investigación. Se ha utilizado como herramienta principal SPSS v.26, aunque también Excel y las propias estadísticas de Google Forms. Se aplicó la prueba Kolmogorov-Smirnov para comprobar la normalidad de los valores, con un nivel de significación mayor a 0.05 . La fiabilidad del cuestionario se ha calculado en ambas fases, a través del alpha de Cronbach. En la primera experiencia (4DLE) el resultado $(\alpha=.737)$ nos ofrece una consistencia adecuada del formulario para confirmar nuestros fines (Hernández, Fernández, \& Baptista, 2003; Bisquerra, 2004). En la segunda fase (INVTIC), con menos preguntas y menos participantes, con una segunda fase de validación, obtenemos un resultado incluso mejor $(\alpha=.825)$.

Tras el análisis descriptivo de los ítems (medias y desviación típica), se aplicó la prueba $t$ de Student, en ambas fases.

Las preguntas del instrumento están referidas a la percepción del alumnado de la Facultad de Educación, antes y después de realizar la práctica grupal de constelaciones multimodales y la valoración de las mismas en su formación docente. De los múltiples conceptos analizados, nos centraremos en los aprendizajes respecto al intertexto 
lector, la competencia literaria, las constelaciones literarias y multimodales, la competencia digital y las narrativas transmedia, porque además de ser los ejes de nuestra propuesta son los que ofrecen resultados más significativos.

\section{Resultados}

Aunque la secuencia didáctica de las constelaciones multimodales y el instrumento utilizado (formularios con pretest y postest) son casi idénticos en las experiencias 4DLE e INVTICUA, preferimos presentar los resultados en dos fases, para demostrar también la evolución de nuestra propuesta.

\section{1. Resultados primera experiencia $4 D L E$}

Las constelaciones de esta propuesta están recogidas en el siguiente blog https://constelacionesmultimodales4dleua19.blogspot.com/, y se centran en los pecados capitales y sus representaciones literarias $\mathrm{y}$ multimodales que conoce nuestro alumnado.

En la tercera tabla recogemos los valores obtenidos en los estadísticos descriptivos del grupo experimental en G1. Estos resultados indican que la percepción del alumnado sobre sus conocimientos, al principio de la secuencia, respecto a conceptos relativos a la Didáctica de la Lengua y la Literatura, es neutra, mostrado un conocimiento superficial de la mayoría de los conceptos que deben trabajarse más detenidamente en esta asignatura, última del área en su formación como maestras. El cuestionario parte de contenidos generales trabajados en cursos anteriores, como competencia literaria, intertexto lector $\mathrm{o}$ educación literaria, elementos centrales en su formación como docentes de literatura. Posteriormente, se centra en contenidos relacionados con la práctica realizada, como las narrativas transmedia o la lectura multimodal, que son los que ocupan principalmente nuestro interés. 
Recordando que 3 es el resultado intermedio de la encuesta, se observa una escasa percepción respecto a sus conocimientos sobre conceptos relativos a la práctica tales como: competencia literaria $(\mathrm{M}=$ $3.24)$; intertexto lector $(\mathrm{M}=3.35)$; narrativas transmedia $(\mathrm{M}=3.67) \mathrm{o}$ constelación multimodal $(\mathrm{M}=3.82)$ con valores medios entre 3 y 4 ( $\mathrm{M}$ $\geq 3.24$ ). Constatamos una elevada dispersión de los datos y una escasa dispersión de las respuestas emitidas (DT $\leq 1.194$ ). Además, el alumnado percibe que no ha recibido suficiente formación sobre qué son las relaciones intertextuales $(\mathrm{M} \geq 3.22$; DT $\leq 0.734)$, contenido central en la asignatura de Didáctica de la Lectura y la Escritura (4DLE).

Estos resultados destacan la importancia y necesidad de instruir en contenidos como la educación literaria (Cerrillo, 2007, Amo \& Núñez Delgado, 2019), la formación lectora y literaria, (Ballester, 2015), el intertexto lector (Mendoza, 2001) y las relaciones intertextuales (Ortiz Ballesteros, 2020) para la enseñanza de la literatura. Además, la propuesta didáctica de las constelaciones incluye nuevos conceptos como la lectura multimodal (Kress, 2010) y las narrativas transmedia (Jenkins, 2003; Scolari, 2013)

\section{Tabla 3}

Resultados descriptivos pretest $1^{a}$ encuesta en G1 4DLE

\begin{tabular}{lll}
\hline Variables del instrumento analizadas & $\mathbf{1}^{\text {a } \text { fase }}$ & \\
\cline { 2 - 3 } & $\mathbf{M}$ & $\mathbf{D T}$ \\
\hline Ítem 8. ¿Soy capaz de definir la competencia literaria? & 3.24 & 1.098 \\
\hline Ítem 10. ¿Soy capaz de definir lo que es el intertexto lector? & 3.35 & 1.194 \\
\hline Ítem 12. ¿Sé qué son las relaciones intertextuales? & 3.22 & 0.734 \\
\hline $\begin{array}{l}\text { Ítem 18. ¿Soy capaz de poner dos ejemplos de relaciones } \\
\text { intertextuales? }\end{array}$ & 3.05 & 0.811 \\
\hline Ítem 20. ¿Sabes lo que son las narrativas transmedia? & 3.67 & 0.873 \\
\hline Ítem 22. ¿Sabes lo que es una constelación multimodal? & 3.82 & 0.804 \\
\hline DT = desviación típica; M = media. Fuente: Elaboración propia & &
\end{tabular}

Aunque los tres primeros ítems señalados reenvían a conocimientos declarativos que no tienen por qué coincidir con el verdadero nivel de conocimiento de las personas encuestadas, se centran en conceptos clave de la asignatura, trabajados en cursos anteriores. El cuarto ya se centra en ejemplos concretos. Podemos 
señalar los resultados de las últimas preguntas (ítems 20 y 22) en sintonía con el resto de respuestas (ítems: 8, 10, 12 y 18), con unos valores descriptivos $(\mathrm{M} \leq 3.99$; DT $\leq 1.194)$ próximos a la opción de respuesta 3 "Ni de acuerdo, ni en desacuerdo" como recoge la anterior tabla. Entendemos la opción 3, intermedia en nuestra escala, como el de conocimiento superficial del tema, sin posibilidad de explicarlo o poner ejemplos. Tales respuestas coinciden con la falta de conocimientos previos respecto a ambos conceptos, por lo que se trata de contenido totalmente nuevo para el alumnado, dato que se confirma en los estadísticos analizados.

Ya que la intervención se centra en la utilidad de las constelaciones multimodales para la mejor comprensión de los conceptos de intertexto lector y relaciones intertextuales, se procede al análisis de los mismos ítems 8, 10,12,18, 20 y 22 en el postest de 4DLE (tabla 4), ya que estas preguntas son claves para valorar la funcionalidad de la secuencia utilizada. El aprendizaje en todos los ítems es evidente si comparamos la tabla 3 con la 4, donde todas las preguntas alcanzan el 4, excepto la 18, que se queda en 3.97 pero también crece significativamente, demostrando la utilidad de la práctica.

\section{Tabla 4}

Variables clave, resultados postest G1 o de referencia en $4 D L E$

\begin{tabular}{lcc}
\multicolumn{1}{c}{ Variables del instrumento analizadas } & \multicolumn{2}{c}{$\mathbf{2}^{\mathbf{a}}$ fase } \\
\cline { 2 - 3 } & $\mathbf{M}$ & $\mathbf{D T}$ \\
\hline Ítem 8 ¿Soy capaz de definir la competencia literaria? & 4.28 & 0.465 \\
\hline Ítem 10. ¿Soy capaz de definir lo que es el intertexto lector? & 4.35 & 0.614 \\
\hline Ítem 12. ¿Sé qué son las relaciones intertextuales? & 4.16 & 0.650 \\
\hline Ítem 18. ¿Soy capaz de poner dos ejemplos de relaciones & 3.97 & 0.948 \\
intertextuales? & & \\
\hline Ítem 20. ¿Sabes lo que son las narrativas transmedia? & 4.49 & 0.789 \\
\hline Ítem 22. ¿Sabes lo que es una constelación multimodal? & 4.33 & 0.689 \\
\hline
\end{tabular}

DT = desviación típica; $\mathrm{M}=$ media. Fuente: Elaboración propia 


\subsection{Análisis comparativo de los grupos 4DLE}

Tras comprobar la normalidad de los datos, se procede a realizar la $t$ de Student para muestras independientes. Se asume también la homogeneidad de las varianzas, tras realizar la prueba $F$ de Levene, con una significancia menor a 0.05 .

Al tener dos grupos en esta experiencia, el objetivo esencial ha sido conocer y valorar si, después de la realización de la práctica de constelaciones multimodales, existen diferencias o no entre ambos grupos. Se analizan los aprendizajes tanto de conceptos clave en la Didáctica de la Lengua y la Literatura: competencia y educación literaria, intertexto lector y relaciones intertextuales; como contenidos propios de la práctica: constelaciones, narrativas transmedia y lectura multimodal. Se formulan las siguientes hipótesis:

a) $H_{0}$ (hipótesis nula): "No existen diferencias significativas entre los ítems analizados relativos al intertexto lector y las narrativas transmedia establecidas en el instrumento de análisis y cada uno de los grupos participantes, con un riesgo alfa de equivocarnos del 0.05 ;

b) $H_{1}$ (hipótesis positiva o alternativa): "Sí existen diferencias significativas establecidas en el instrumento de análisis y el grupo de pertenencia de los participantes, con un riesgo alfa de equivocarnos del $0.05 "$.

\section{Tabla 5}

$t$ de Student del G1 experimental y del G2 control analizados en 4DLE

\section{$\mathbf{2}^{\mathbf{a}}$ fase}

\begin{tabular}{|c|c|c|c|c|c|c|}
\hline \multirow[t]{2}{*}{ Var. } & \multicolumn{2}{|l|}{ G 1} & \multicolumn{2}{|l|}{ G 2} & \multirow[b]{2}{*}{$t$} & \multirow[b]{2}{*}{$\mathbf{p}$} \\
\hline & $\mathbf{M}$ & DT & $\mathbf{M}$ & DT & & \\
\hline Ítem 8 & 4.28 & 0.465 & 4.01 & 0.571 & -1.903 & 0.049 \\
\hline Ítem 10 & 4.35 & 0.614 & 3.82 & 0.670 & -1.700 & 0.031 \\
\hline Ítem 12 & 4.16 & 0.650 & 4.06 & 0.778 & -1.413 & 0.044 \\
\hline Ítem 18 & 3.97 & 0.948 & 3.13 & 1.050 & -0.150 & 0.881 \\
\hline Ítem 20 & 4.49 & 0.789 & 3.78 & 0.917 & -1.603 & 0.022 \\
\hline Ítem 22 & 4.33 & 0.689 & 3.82 & 0.921 & -2.338 & 0.021 \\
\hline
\end{tabular}

Var. = variable; $\mathrm{DT}=$ desviación típica; $\mathrm{M}=$ media; $\mathrm{G} 1=$ experimental $\mathrm{o}$ de referencia; G2= control o diagnóstico. Fuente: Elaboración propia 
Podemos señalar diferencias relevantes en el conjunto global de datos en función de cada grupo. En un análisis detallado observamos diferencias estadísticamente significativas en seis de las cuestiones planteadas. El grupo de referencia $(\mathrm{G} 1 ; 4.28)$ percibe que sí es capaz de definir la competencia literaria (ítem 8) de una forma más detallada que el grupo de control (G2 4.01). Asimismo, encontramos diferencias respecto a los conceptos de intertexto lector (ítem 10: G1 media=4:35 frente a G2: 3.82) y las relaciones intertextuales (ítem 12 G1 media $=4.16$ frente a G2: 4.06), ejes esenciales para relacionar los diversos elementos que componen una constelación. Obviamente, las diferencias más destacadas las encontramos en el ítem 20 "qué son las narrativas transmedia" (G1, media $=4.49$ frente a $\mathrm{G} 2: 3.78)$ y en el ítem 22 "sobre constelación multimodal" (G1, media=4.33 frente a G2: 3.82) ya que son el centro de la actuación en el grupo experimental. Por un lado, se comprueba la importancia para conceptos clave de la DLL (ítems 8, 10, 12 o 18) y por otro, se reconocen los contenidos específicos trabajados en la secuencia (ítems 20 y 22).

Sin embargo, en el análisis del resultado algunas cuestiones (18) no muestran diferencias significativas. El alumnado de G1 muestra una percepción mejor para elaborar ejemplos de relaciones intertextuales (ítem 18) frente a $\mathrm{G} 2$, pero la $t$ nos señala que las diferencias no son significativas. No hay una desigualdad relevante frente al resto de preguntas que sí la tienen. Posiblemente se debe a que este concepto sí que se trabaja en las prácticas alternativas del G2 y en los materiales teóricos del programa de la asignatura, comunes a G1 y G2.

\subsection{Resultados de la segunda experiencia INVTICUA}

Las constelaciones de la Universidad de Alicante de esta segunda fase están recogidas en el siguiente blog https://ecostransmedia2020.blogspot.com/, junto con las propuestas de la Universidad de Murcia, y se centran en constelaciones sobre personajes de la literatura infantil tradicional. 
Las personas que han participado en la propuesta didáctica son un total de 34 , excluyendo posteriormente un caso al no completar uno de los dos cuestionarios (pretest y postest) (33). Esta exclusión no altera la representatividad de la muestra por debajo del 95\%. Este grupo tiene mayor edad y formación universitaria, porque están en el Máster de Secundaria. Recordemos, como ya hemos comentado en otras ocasiones (Rovira-Collado, 2019) que este alumnado tiene muchos más conocimientos literarios, porque tienen en su mayoría formación filológica frente al alumnado del grado de maestro. Esta es una variable independiente fundamental, otro motivo para diferenciar los resultados de las dos experiencias. Aunque el conocimiento literario es mayor, las destrezas didácticas están en pleno desarrollo (Ramos, 2019).

Se identifican 5 variables relacionadas directamente con las categorías analizadas en la fase anterior. Son las siguientes: INTX: intertexto lector; CLIT: competencia literaria; CONS: constelaciones literarias. CDIG: competencia digital; y NT: narrativas transmedia.

El análisis sobre las 5 variables relacionadas con las preguntas de escala Likert de 5 puntos muestra diferencias estadísticamente significativas en 4 de las 5 variables, reflejado en los datos (Tabla 6) que recogen la progresión en el periodo mediante el análisis de la prueba $t$ de muestras relacionadas.

\section{Tabla 6}

Resultados de la prueba t para muestras relacionadas en INVTIC

\begin{tabular}{lccccc}
\hline Variable & Media antes & Media después & $t$ & $g l$ & $P$ \\
\hline INTX & 3,91 & 4,18 & $-1,43$ & 33 & .163 \\
CLIT & 3,74 & 4,41 & $-3,44$ & 33 & .002 \\
CONS & 1,76 & 3,94 & $-8,42$ & 33 & .000 \\
CDIG & 3,53 & 4,29 & $-4,04$ & 33 & .000 \\
NT & 1,56 & 4,26 & $-14,15$ & 33 & .000 \\
\hline
\end{tabular}

$\mathrm{N}=33$. Variables: INTX: intertexto lector. CLIT: competencia literaria. CONS: constelaciones literarias. CDIG: competencia digital. NT: narrativas transmedia.

Fuente: Martínez-Carratalá \& Hernández Delgado (2020)

En esta segunda experiencia, destacamos que los conceptos que muestran un mayor progreso y diferencias estadísticamente 
significativas están relacionados con el aprendizaje de conceptos clave de este proyecto, como son las narrativas transmedia o las constelaciones literarias, a través de su adaptación multimodal. El dato más significativo está relacionado con los aprendizajes sobre narrativas transmedia (NT) que se desarrollan desde la presentación de la práctica $(t$ (33) $=-14,15, p=.000)$ entre los resultados obtenidos antes de la secuencia didáctica, sin la presentación del concepto $(\mathrm{M}=1,56 ; \mathrm{DS}=$ .89) y después, con la presentación de las constelaciones y relatos $(\mathrm{M}=$ $4,26 ; \mathrm{DS}=.75)$.

Otro elemento importante es el término de intertexto lector. Aunque es un concepto que demuestra una evolución en los resultados obtenidos antes $(\mathrm{M}=3,91 ; \mathrm{DS}=.79)$ y después $(\mathrm{M}=4,18 ; \mathrm{DS}=.90)$ de la práctica, no resulta estadísticamente significativo. Para finalizar, la evolución de la comprensión de la competencia digital y su importancia es otro de los elementos que resultan estadísticamente significativos ( $t$ (33) $=-4,04, p=.000$ ) en esta segunda propuesta. Recordemos que la asignatura donde se desarrolla (INVTIC), está muy relacionada con la tecnología educativa, mientras que la competencia literaria se trabaja en otras de esta titulación.

En esta segunda prueba, además se realizó un análisis descriptivo de los resultados, contabilizando los elementos recogidos en las constelaciones, a través de la segunda muestra (constelaciones bibliométricas) donde se incluye la referencia completa de cada elemento incluido en la imagen de la constelación. Cada grupo debía recoger, como mínimo, 15 referencias literarias y 15 multimodales. En la tabla 7 vemos el total de referencias de cada constelación realizada, identificada con el/la protagonista de la constelación. 


\section{Tabla 7}

Elementos recogidos en las constelaciones bibliométricas INVTIC

\begin{tabular}{lcccc}
\hline Cuento & $\begin{array}{c}\text { Referencias } \\
\text { literarias }\end{array}$ & $\begin{array}{c}\text { Referencias } \\
\text { multimodales }\end{array}$ & Total & Diferencia \\
\hline Blancanieves & 21 & 27 & 48 & -6 \\
$\begin{array}{l}\text { Gato con botas } \\
\text { Cenicienta }\end{array}$ & 16 & 15 & 31 & +1 \\
$\begin{array}{l}\text { Bella durmiente } \\
\text { El soldadito }\end{array}$ & 24 & 23 & 47 & +1 \\
plomo & 32 & 35 & 67 & -3 \\
El patito feo & 16 & 40 & 56 & -24 \\
TOTALES & 27 & 70 & 97 & -43 \\
\hline
\end{tabular}

Fuente: Elaboración propia

Los resultados reflejan el trabajo de los diferentes grupos y su documentación también muestra las diferentes interpretaciones que han realizado del cuento original y sus derivaciones. Estas se estructuran en representaciones exclusivamente relacionadas con la literatura (desde el hipotexto a renovaciones en la Literatura Infantil y Juvenil o la poesía). El segundo grupo se ha agrupado como referencias multimodales, pero incluyen también otras expresiones artísticas (esculturas o pinturas), producciones audiovisuales (cine y series), musicales (canciones $\mathrm{o}$ álbumes) y multimedia (como videojuegos) hasta su presencia en diferentes plataformas, redes sociales, campañas publicitarias, aplicaciones de móvil, productos de belleza y diferentes productos publicitarios.

Las estrategias de búsqueda se han centrado en diferentes criterios, pero han combinado las alusiones hipertextuales (cuando la reescritura está basada en el hipotexto) y referencias intertextuales (mucho más complejas al ser reinterpretaciones del original que prescinden de la alusión directa). Otro aspecto relevante ha sido la manera en que los distintos grupos han representado sus constelaciones mediante imágenes. La mayoría de los grupos ha optado por la opción mostrada en el blog que servía de guía, mientras que también se han dado otras modalidades de presentación como Prezi (La bella durmiente) o la trasmodalización (cambio del modo de representación) por parte del grupo que tenía asociado el cuento El patito feo que ha 
realizado una presentación en vídeo compartido en la plataforma YouTube.

La importancia de esta fase, más que un análisis cuantitativo de las referencias, se centra en la capacidad del alumnado participante para comprobar la presencia de los cuentos populares en el ámbito literario y cultural. En ese momento crean 6 constelaciones multimodales en los que se comprueban las diferentes reinterpretaciones que efectúa cada grupo. La mayoría de los grupos se sirve de los diferentes elementos destacables del cuento para establecer relaciones intertextuales con diferentes representaciones.

Este posterior análisis queda pendiente de realizar en la primera experiencia, centrada en los pecados capitales.

\section{Discusión y conclusiones}

Los aprendizajes confirmados por la investigación en ambos proyectos han mostrado diferencias estadísticamente significativas entre las medidas antes y después de las variables (INVTIC) y entre el grupo experimental y de control (4DLE). Por lo tanto, se rechaza la hipótesis nula planteada en la investigación. De manera global, la propuesta de constelaciones multimodales ha sido apropiada para la formación docente y ha servido para confirmar aprendizajes del área (intertexto lector, competencia literaria), junto la adquisición de nuevos conceptos (lectura multimodal, narrativas transmedia).

Podemos señalar diferencias entre ambos grupos, como, por ejemplo, que los conceptos más literarios eran ya más conocidos en el alumnado del máster de secundaria, mientras que los más digitales son novedosos en ambos grupos. De esta manera, hay que destacar las variables relacionadas con la percepción del aprendizaje sobre conceptos como constelaciones literarias, narrativas transmedia y lectura multimodal. 
Por estos motivos reiteramos la utilidad del uso del formato de las constelaciones literarias (Jover, 2009) y multimodales (RoviraCollado, 2019) en el aula. Estas han permitido un trabajo de documentación exhaustiva respecto a los pecados capitales o a personajes de los cuentos de referencia como un género fundamental por la universalidad de sus representaciones (Zipes, 2006), confirmando que existen infinidad de referentes en ambas experiencias. Sin duda, este formato posibilita la activación del intertexto lector (Mendoza, 2001) de los diferentes grupos de trabajo, siendo a su vez una actividad socializada por sus diferentes componentes.

En conjunto, ambas secuencias han permitido el desarrollo de una competencia literaria (Cerrillo, 2007; Lluch, 2003) en el alumnado universitario que les posibilita como futuras mediadoras y mediadores al acceso a los diferentes niveles de interpretación de una obra artística. Ambas temáticas permiten el reconocimiento de valores y son vehículo de transmisión de conocimientos más allá de los literarios. Entre estos, no solo los referidos a sus capacidades y destrezas en su competencia digital docente (INTEF, 2017), incorporando la lectura de hipertextos digitales (Landow, 2009) mediante el análisis de estos de manera crítica y consciente (Mendoza, 2012). A su vez, la comprensión de esta multimodalidad (Kress \& Selander, 2012; Bombini \& Martínez, 2018) se debe completar con las capacidades de comprensión de estas en su recepción (Unsworth, 2014).

Aunque son dos recorridos formativos distintos, entre el alumnado del Grado de Primaria y el Máster de Secundaria encontramos lectores literarios competentes (Cerrillo, 2016) al enfrentarse a la navegación hipertextual de manera estructurada en la secuencia didáctica. La primera propuesta 4DLE, centrada en los pecados capitales, nos ha permitido comprobar cómo el alumnado ha sido capaz de relacionar la lectura de la Divina Comedia de Dante, el Lazarillo de Tormes o el análisis de El Jardín de las Delicias del Bosco con representaciones contemporáneas de los pecados capitales en series de televisión, cómics, relatos de literatura infantil y juvenil o incluso su relación con las redes sociales que manejan a diario, generando narrativas híbridas (Encabo, Hernández y Sánchez, 2019). 
La pervivencia de las narrativas de tradición oral también aparece en el análisis de las constelaciones y relatos INVTIC, donde hay un cambio de valores a través de las reinterpretaciones hipertextuales y referencias intertextuales en el ámbito literario (Colomer \& Olid, 2009; Juri, 2010). En contrapartida, este segundo grupo refleja en sus creaciones la transgresión contra los valores representados en las producciones Disney y la importancia que han tenido estas en el desarrollo de la competencia enciclopédica como futuros mediadores en el aula (López-Valero, Hernández-Delgado \& Encabo, 2017). Esta intertextualidad multimedia (Kinder, 1991) se ha transgredido en sus relatos que han optado por diferentes estrategias en sus reelaboraciones (Genette, 2001) y se han identificado diferentes mecanismos de relaciones hipertextuales e intertextuales en sus relaciones en línea con el análisis (Amo, 2019) y las diferentes producciones en plataformas dedicadas a la producción de reelaboraciones por parte de escritores digitales que reinterpretan sus narrativas y personajes (García-Roca \& Amo, 2019).

Finalmente, se ha comprobado que la creación de los epitextos digitales de 4DLE y los relatos multimodales INVTIC mejora la capacidad de creatividad de los participantes, completando la secuencia didáctica mediante un diseño narrativo multiplataforma (Scolari, 2019) que emplea la multimodalidad de representaciones y relaciones intertextuales con una variedad de recursos que propician una mejora en su competencia comunicativa y digital (Ramos-Sabaté, 2010).

La investigación en el ámbito de la educación literaria persigue una formación docente integral e interdisciplinar, que incorpore a sus conocimientos literarios una concepción pedagógica amplia (Núñez, Cea \& Silva, 2019). Mediante la secuencia didáctica empleada, no solo se ha incrementado la capacidad para la lectura hipertextual (tanto literaria como digital) sino que ha ampliado las capacidades y saberes de los participantes con la inclusión de la creación de constelaciones y textos literarios y multimodales (Perry, 2020). 
Esta propuesta didáctica, amplía las investigaciones previas y refleja la importancia de la competencia digital en el área de literatura, más allá de las destrezas tecnológicas para su elaboración, con la inclusión de aspectos éticos y la identificación de los valores de ambas temáticas trabajadas.

Como limitaciones de esta investigación se destaca la situación de excepcionalidad en el desarrollo de la segunda propuesta, que finalizó la práctica durante el confinamiento provocado por la pandemia de Covid-19 entre marzo y junio de 2020. A pesar de todas las dificultades, el carácter digital de ambas propuestas y la necesidad del trabajo cooperativo inscrita en el diseño de esta práctica transmedia (Castañeda, 2021) reafirma la utilidad de la misma.

En conclusión, el aprendizaje mediante la práctica creativa de los participantes ha permitido investigar los mecanismos de recepción e interpretación del enorme conjunto de obras recopilados en las constelaciones (Martínez-Carratalá \& Hernández Delgado, 2020). Adicionalmente, el relato multimodal supone una renovación de las creaciones digitales en otras plataformas e incorpora elementos audiovisuales que permiten un análisis de sus alusiones, modos y formatos que complementa la practica literaria.

\section{Referencias bibliográficas}

Ambròs Pallarès, A. (2020). Cinema, Transmèdia i Educació: relats en pantalla. Revista de Innovació i Recerca en Educació, 13(1) doi: https://doi.org/10.1344/reire2020.13.128644.

Amo Sánchez Fortún, J. M. (2019). La mutación cultural: estudios sobre la lectura digital. En J. M. Amo Sánchez-Fortún (Coord.), Nuevos modos de lectura en la era digital (pp.15-40). Síntesis.

Amo Sánchez-Fortún, J.M., \& Núñez Delgado, P. (Eds.) (2019) Lectura y educación literaria. Nuevos modos de leer en la era digital. Octaedro. 
Arbonés Solá C., Mendoza Fillola, A., Muñoz Gordillo, M. S., Prats Ripoll, M., \& Sanahuja Yll E. (2015). Textualidades multimodales, conectividad y redes 2.0 en el aula: sobre la educación literaria y el profesorado en formación. En J. M. Amo, O. Cleger \& A. Mendoza, A. (2015). Redes hipertextuales en el aula (pp.97-120). Octaedro.

Argibay, J.C. (2009). Muestra en investigación cuantitativa. Subjetividad y Procesos Cognitivos, 13(1), 13-29.

Ballester, J. (2015). La formación lectora y literaria. Graó.

Bisquerra, R. (coord.) (2004). Metodología de la investigación educativa. La Muralla.

Bombini, G., \& Martínez, N. V. (2018). Lectura, escritura y "nuevas tecnologías": un desafío a la imaginación didáctica. Textos de didáctica de la lengua y la literatura, 80, 7-13.

Caro-Valverde, M.T. (2019). El clásico, irreductible. Historia de vida en didáctica de la creación literaria mutimodal. Tejuelo, 29, 254-274. doi: https://doi.org/10.17398/1988-8430.29.245.

Castañeda, L. (2019). Formación inicial del profesorado en el uso educativo de la tecnología, una propuesta curricular. Quaderns Digitals, 89. http://www.quadernsdigitals.net/index.php?accionMenu=hemeroteca. Vi sualizaArticuloIU.visualiza\&articulo_id=11517.

Castañeda, L. (2021). Una experiencia de diseño de una tarea de evaluación sumativa en formato transmedia para formación inicial de profesorado. RIED. Revista Iberoamericana de Educación a Distancia, 24(2), (versión preprint). doi: https://doi.org/10.5944/ried.24.2.29148.

Cerrillo, P. (2016). El lector literario. Fondo de Cultura Económica.

Cerrillo. P. (2007). Literatura infantil y juvenil y educación literaria: hacia una nueva enseñanza de la literatura. Octaedro.

Colomer, T., \& Olid, I. (2009). Princesas con tatuaje: las nuevas caras del sexismo en la ficción juvenil. Textos de Didáctica de la Lengua y la Literatura, 51, 55-67.

De Benito, B., \& Salinas, J. (2016). La Investigación Basada en Diseño en Tecnología Educativa. Revista Interuniversitaria de Investigación en Tecnología Educativa. doi: https://doi.org/10.6018/riite2016/260631. 
Del Moral-Barrigüete, C., \& Molina-García, M. J. (2018). Diseño y validación de un cuestionario sobre hábitos lectores en futuros maestros durante su etapa preuniversitaria. Revista de Educación de la Universidad de Granada, 25. doi: 10.30827/reugra.v25i0.100.

Encabo, E., Hernández, L. \& Sánchez, G. (2019). La literatura infantil y las narrativas híbridas. Canon, modos de pensamiento y educación. Contextos educativos, 23, 199-212. doi: https://doi.org/10.18172/con.3496.

García Roca, A., \& De Amo, J. M. (2019). Jóvenes escritores en la red: un estudio exploratorio sobre perfiles de Wattpad. Ocnos. Revista de Estudios de Lectura, 18 (3), 18-28. doi: https://doi.org/10.18239/ocnos_2019.18.3.1968.

Garvis, S. (2015). Narrative Constellations. Exploring Lived Experience in Education. Sense publishers, University of Gothenburg.

Genette, G. (2001). Umbrales. Siglo XXI.

Gómez, I. M., \& Ruiz, M. (2018). Interdisciplinariedad y TIC: Nuevas Metodologías Docentes aplicadas a la enseñanza superior. Pixel-Bit, 52, 67-80. doi: https://doi.org/10.12795/pixelbit.2018.i52.05

Gómez-Trigueros, I. M., Ruiz-Bañuls, M., \& Ortega-Sánchez, D. (2019). Digital Literacy of Teachers in Training: Moving from ICTs (Information and Communication Technologies) to LKTs (Learning and Knowledge Technologies). Education Science, 9, (274). doi: https://doi.org/10.3390/educsci9040274.

Hernández Ortega, J. (2019). Representaciones transmedia en entornos de lectura analógica. Tecnología, Ciencia y Educación, 14, 536.

https://tecnologia-cienciaeducacion.com/index.php/TCE/article/view/329.

Hernández Ortega, J., \& Rovira-Collado, J. (2020). Diseño de proyectos transmedia para la Educación Literaria en el aula de Educación Secundaria. Revista De Estudios Socioeducativos. ReSed, 8, 80-98. https://revistas.uca.es/index.php/ReSed/article/view/5633.

Hernández, R., Fernández, C., \& Baptista, P. (2003). Metodología de la Investigación. Mc.Graw Hill.

INTEF (2017). Marco Común de Competencia Digital Docente 2017. MECD. https://aprende.intef.es/sites/default/files/201805/2017_1020_Marco-Com\%C3\%BAn-de-Competencia-DigitalDocente.pdf. 
Jenkins, H. (2003). Transmedia Storytelling. Technology Review. https://www.technologyreview.com/s/401760/transmediastorytelling/.

Jenkins, H. (2015). Cultura transmedia: la creación de contenido y valor en una cultura en la red. Gedisa.

Jover, G. (2007). Un mundo para leer. Octaedro.

Jover, G. (2009). Constelaciones literarias. Sentirse raro. Miradas sobre la adolescencia. Junta de Andalucía.

Juri, S. (2010). Sobre los libros de juego intertextual con los cuentos populares. En A. Mendoza Fillola \& C. Romea (Eds.), El lector ante la obra hipertextual (pp.43-56). Horsori Editorial.

Kinder, M. (1991). Playing with power in movies, television, and video games: from Muppet Babies to Teenage Mutant Ninja Turtles. University of California Press.

Kress, G. R. (2010). Multimodality: A Social Semiotic Approach to Contemporary Communication. Routledge.

Kress, G. \& Selander, S. (2012). Multimodal design, learning and cultures of recognition. Internet and Higher Education, 15(4), 265268. doi: https://doi.org/10.1016/j.iheduc.2011.12.003.

Landow, G. P. (2009). Hipertexto 3.0. La teoría crítica y los nuevos medios en una época de globalización. Paidós.

Larrañaga, E., \& Yubero, S. (2015). Evaluación de las estrategias metacognitivas de comprensión de textos narrativos. Ocnos. Revista de Estudios de Lectura, 14. doi: https://doi.org/10.18239/ocnos_2015.14.02.

Leeuwen, T., \& Jewitt, C. (2001). The Handbook of Visual Analysis. SAGE.

Lluch, G. (2003). Análisis de narrativas infantiles y juveniles. Ediciones de la Universidad de Castilla-La Mancha.

Lluch, G., Tabernero-Sala, R., \& Calvo-Valios, V. (2015). Epitextos virtuales públicos como herramientas para la difusión del libro. El profesional de la información, 24(6), 797-804. doi: http://dx.doi.org/10.3145/epi.2015.nov.11.

López Valero, A., Hernández-Delgado, L. \& Encabo, E. (2017). El concepto de Literatura infantil. Un estudio de caso con maestros en formación inicial. Ocnos. Revista de Estudios de Lectura, 16 (2), 37-49. doi: https://doi.org/10.18239/ocnos_2017.16.2.1400. 
Martínez-Carratalá, F. A. (2020). Caperucita transmedia: uso de blogs de LIJ para el desarrollo crítico de la competencia literaria y digital en la formación docente. En R. Roig-Vila (Coord.), Redes de Investigación e Innovación en Docencia Universitaria. Volumen 2020 (pp. 293-302). Universidad de Alicante. http://rua.ua.es/dspace/handle/10045/110037.

Martínez-Carratalá, F. A., \& Hernández Delgado, L. (2020) Desarrollo de la competencia digital y literaria en la formación del profesorado a través de narrativas multimodales de tradición oral. En R. Roig-Vila (Ed.). La docencia en la Enseñanza Superior. Nuevas aportaciones desde la investigación e innovación educativas (pp. 719728). Octaedro.

McGrath, J. (1986). Groups Interaction and Performance. Prentice Hall.

Mendoza, A. (2001). El intertexto lector. Ediciones de la Universidad de Castilla-La Mancha.

Mendoza, A. (2012). Leer hipertextos. Del marco hipertextual a la formación del lector literario. Octaedro.

Mendoza, A., Castro, C. R., Gordillo, S. M., \& Gómez, M. A. H. (2016). Propuestas y experiencias sobre estructuras hipertextuales literarias en el aula. Octaedro.

Mendoza, A., \& Cerrillo, P. (2013). Intertextos: aspectos sobre la recepción del discurso artístico. Universidad de Castilla la Mancha.

Mendoza, A., \& Romea, C. (2010). El lector ante la obra hipertextual. Horsori.

Núñez Sabarís, X., Cea Álvarez, A. M ${ }^{a}$. \& Silva Dias, A. (2019). Literatura por tareas y gamificación: novela policíaca y geografías culturales. Tejuelo 30, 261-288. doi: https://doi.org/10.17398/1988-8430.30.26.

Ortiz Ballesteros, A. M. (2020). La intertextualidad en las revistas científicas de didáctica de la lengua y la literatura. Tejuelo, 32, 83-116. doi: https://doi.org/10.17398/1988-8430.32.83.

Paladines-Paredes, L. \& Aliagas, C. (2021). Videoreseñas de booktubers como espacios de mediación literaria. Ocnos. Revista De Estudios Sobre Lectura, 20(1), 38-49. doi: https://doi.org/10.18239/ocnos_2021.20.1.2489. 
Paredes Labra, J., Guitert Catasús, M., \& Rubia Avi, B. (2015). La innovación y la tecnología educativa como base de la formación inicial del profesorado para la renovación de la enseñanza. Relatec, 14(1), 101-114. doi: https://doi.org/10.17398/1695-288X.14.1.101.

Perry, M. S. (2020). Multimodal Engagement through a Transmedia Storytelling Project for Undergraduate Students. Gema Online Journal of Language Studies, 20(3), 19-40. doi: https://doi.org/10.17576/gema-2020-2003-02.

Prendes Espinosa, M. P., Gutiérrez Porlán, I., \& Castañeda Quintero, L. (2019). La capacitación en competencia digital docente del profesorado. En M. Gisbert Cervera, V. Esteve-González, V. \& J. L. Lázaro Cantabrana (Eds.) (2019), ¿Cómo abordar la educación del futuro? Conceptualización, desarrollo y evaluación desde la competencia digital docente (pp. 217-234). Octaedro.

Ramos, J. M. (2019). La creación de una exposición virtual de poetas clásicos catalanes en el aula de secundaria. Tejuelo, 29, 187-216. doi: https://doi.org/10.17398/1988-8430.29.187.

Ramos Sabaté, J. M. (2010). Lectores re-creando el texto. En A. Mendoza \& C. Romea (Eds.), El lector ante la obra hipertextual (pp.129-136). Horsori Editorial.

Romea Castro, C. (2008). ¿Cómo leemos en la era de Bill Gates? Una mirada postmoderna a los textos. En A. Mendoza (Coord.). (2008), Textos entre textos: las conexiones textuales en la formación literaria. (pp.91-104). Horsori Editorial.

Romero, M. F., Ambrós, A., \& Trujillo, F. (2020). Hábitos lectores de los adolescentes en un ecosistema llamado escuela: factores determinantes en estudiantes de educación secundaria. Investigaciones Sobre Lectura, 13, 18-34. doi: https://doi.org/10.37132/isl.v0i13.295.

Rovira-Collado, J. (2019). Clásicos literarios en constelaciones multimodales. Análisis de propuestas de docentes en formación. Tejuelo, 29, 275-312. doi: https://doi.org/10.17398/1988-8430.29.275.

Scolari, C. (2013). Narrativas transmedia. Cuando todos los medios cuentan. Deusto.

Scolari, C. (2018). Alfabetismo Transmedia en la nueva ecología de los medios. Libro Blanco. H2020 Transliteracy Project. Universitat

https://repositori.upf.edu/handle/10230/33910. 
Scolari, C. (2019). Transmedia Is Dead. Long Live Transmedia! (Or Life, Passion and the Decline of a Concept). LIS. Letra. Imagen. Sonido. Ciudad Mediatizada, 11(20), 69-92. https://repositori.upf.edu/handle/10230/45898.

Unsworth, L. (2014). Multimodal reading comprehension: curriculum expectations and large-scale literacy testing practices. Pedagogies, 9(1), 26-44. doi: https://doi.org/10.1080/1554480X.2014.878968.

Vicente-Yagüe Jara, M. I. (2014). Estudio y valoración de un modelo didáctico intertextual literario-musical. Una investigación en la Región de Murcia. Revista De Investigación Educativa, 32(2), 513-530. doi: https://doi.org/10.6018/rie.32.2.165821.

Zipes, J. (2006). Why fairy tales stick. Routledge. 
Tejuelo, $\mathrm{n}^{\circ} 34$ (2021), págs. 111-142. Intertextualidad y multimodalidad en constelaciones transmedia ...

142 | Pág in a 